Voix et Images

voixetimages

\title{
Réginald Hamel, la Louisiane créole
}

\section{Robert Lahaise}

Volume 10, numéro 3, printemps 1985

André Major

URI : https://id.erudit.org/iderudit/200527ar

DOI : https://doi.org/10.7202/200527ar

Aller au sommaire du numéro

\section{Éditeur(s)}

Université du Québec à Montréal

\section{ISSN}

0318-9201 (imprimé)

1705-933X (numérique)

Découvrir la revue

Citer ce compte rendu

Lahaise, R. (1985). Compte rendu de [Réginald Hamel, la Louisiane créole]. Voix et Images, 10(3), 204-206. https://doi.org/10.7202/200527ar d'utilisation que vous pouvez consulter en ligne.

https://apropos.erudit.org/fr/usagers/politique-dutilisation/ 


\title{
Réginald Hamel, la Louisiane créole'
}

\author{
par Robert Lahaise, Université du Québec à Montréal
}

Comme me le racontait un ami interrogé par un étudiant quant à l'Angola: «... l'Angola, je savais que c'était en Amérique centrale, mais à part ça...» Mais à part ça, madame la marquise, il en est sans doute ainsi pour nous, Québécois francophones, quant à la Louisiane.

Lointaine contrée aux relents exotiques qui servit d'arrière-scène aux romans feuilletons où pullulaient alligators et noires à la peau moite dans de luxuriantes plantation, la Louisiane nous apparaît encore comme étant la terre d'élection de notre cousin Zacharie Richard et celle aussi des lupanars odorants de New Orléans..

Mais... si on a fait un peu d'histoire, on se rappellera que les grands du régime français, Cavelier de La Salle et d'Iberville, ces enjambeurs de continent, y établirent la présence française qui s'étendit bientôt sur le tiers des États-Unis actuels, du sud des Grands Lacs jusqu'au Golfe du Mexique. Et qu'un certain Law fit miroiter cet Eldorado aux yeux d'une France fiévreuse qui s'était terriblement ennuyée durant les dernières années du règne du grand Louis - qui, comme tout grand qui se respecte, avait hérité d'un pays puissant pour le laisser ruiné - grand Louis dis-je, père de la Louisiane. Quant à nous, Québécois, on se souvient que ladite Louisiane, lors de la Défaite de 1760, se promena alors magiquement de l'Espagne à Napoléon, pour finalement aboutir aux États-Unis, en se rétrécissant toujours comme une peau de chagrin.

Et si on a fait un peu de littérature, on aura peut-être lu l'Histoire littéraire de l'Amérique française d'Auguste Viatte pour constater qu'au XIXe siècle, à quelque 3000 kilomètres au sud de la vallée du Saint-Laurent, il y avait surabondance de publications françaises. Mais parce que cet essai de Viatte a été imprimé en France, il y a une trentaine d'années, son actuel rayonnement doit sans doute être assez mince. En somme une triste et quasi totale ignorance pour cette terre de soleil et de bayous où, dit-on, des Acadiens devinrent Cajuns.

Voilà pourquoi il faut remercier Réginald Hamel qui, dans un livre édité au Québec et une optique bien de chez nous, nous fournit, relativement aux études antérieures, un apport triplement nouveau. Tout d'abord, une chronologie (année par année!) de la Louisiane depuis 1762 (date où la France cédait secrètement ce territoire à l'Espagne) jusqu'à 1900 (au moment où la Louisiane francophone entre dans l'ère qu'il faut bien qualifier de folklorique). Tout y passe, tant du point de vue politique - aussi bien aux États-Unis, qu'en Espagne, en France, en Angleterre, au Canada (pour autant qu'il existe des rapports entre ces pays et la Louisiane) - que littéraire et social. Le texte est agrémenté de nombreuses photographies, et plus particulièrement des cartes américaines de l'époque, nous montrant le morcellement continu। du territoire louisianais. En outre, trois tableaux, l'un des 
gouverneurs et des commissaires-ordonnateurs sous le Régime français, un second des gouverneurs espagnols de 1762 à 1803 et enfin un autre, des gouverneurs louisianais de 1804 à 1900 , permettent de bien situer chacun des personnages ou événements dans son contexte. Enfin on trouve, en appendice, une chronologie commentée des centaines de périodiques fondés sur ce territoire depuis 1794 jusqu'à 1899 .

Second apport: l'anthologie de plus de quatre cents pages. D'une part, des textes politico-économiques, depuis la prise de possession du territoire, en 1682, par Cavelier de La Salle, jusqu'à la neuvième constitution, en 1921, avec les articles relatifs aux francophones qui ne forment plus à cette date que $2 \%$ de la population! D'autre part, une anthologie littéraire - semblable à celle de «notre» James Huston au milieu du XIX e siècle - où Hamel, délaissant l'art pour l'art, recherche le texte pour son efficacité historico-sociale. Ayant dépouillé d'innombrables périodiques, il en extrait des articles particulièrement percutants, tel, entre autres, celui d'Eugène Dumez, intitulé "Les apôtres de l'égalité», paru dans le Meschacébé de 1868, où l'on constate que si les principes du Yankee se claironnent bien, la pratique plus réaliste rejette aux oubliettes tout ce qui n'est pas WASP (White Anglo Saxon Protestant) (pp. 383-397). Une fois de plus, la littérature s'avère fille de l'histoire, et Auguste Lussan, dans sa pièce «Les martyrs de la Louisiane» (jouée au Théâtre Français de la Nouvelle-Orléans en 1839 - et non en 1938 tel qu'indiqué au centre de la page 536), ne peut que soupirer, après l'abandon des Louisianais par la France de Choiseul et de son maître dit le «Bien-Aimé»:

«Français hier encor, vous n'avez plus de nom!»

Outre cet aspect socio-littéraire, le linguiste y trouvera également son compte, Alfred Mercier ayant traduit en 1890 quelques Fables d'Ésope en créole louisianais (398-405). À remarquer que, contrairement à beaucoup d'anthologies où l'on ne retrouve guère plus que des documents, Hamel, pour sa part, introduit et commente méticuleusement et amoureusement chacun des extraits, ne fournissant pas moins de son propre cru d'érudition plus de la moitié du texte de ladite anthologie.

Pour compléter ce triptyque que nous donne Hamel avec sa Louisiane créole, venons-en à ce qui me semble le point le plus important: la «louisianisation" d'une population. Totalement française en 1760 , elle ne l'est plus qu'à $60 \%$ en 1812 , et $2 \%$ en 1921 (p. 319). Comment peut-on en arriver à un aussi magnifique résultat? en votant non - qui signifie oui - au Référendum! Eh oui, nom d'un nom, nous avons bien ouï, non? Mais revenons à nos Louisianais, à nos Charles-Étienne-Arthur Gayarré (1805-1895), auteur d'une importante Histoire de la Louisiane (1846-1847), et qui, devenu juge, n'écrit plus qu'en anglais, ou encore Bernard Marigny de Mandeville qui, en 1855, affirme que Napoléon III «se ferait un plaisir (... d'envoyer en Louisiane) cent mille zouaves pour défendre le catholicisme» (p. 369).

Et si d'aucuns considèrent ce rapprochement avec le Québec mesquinement nationaliste et d'un chauvinisme digne d'un Péquiste à ses heures 
fastes, laissons la parole - comme le fait Hamel (p. 47) - à un célèbre Lord, Durham en l'occurrence, auteur d'un non moins célèbre Report on the $A f$ fairs of British North America:

Dans l'histoire de l'État de la Louisiane, dont la population et les lois étaient françaises au temps de la cession à l'Union américaine, on trouve un exemple mémorable de la manière selon laquelle des institutions parfaitement égales et populaires peuvent effacer les distinctions de race sans désordre ou oppression, et avec à peine un peu plus d'animosité que dans les pays libres sujets aux conflits habituels des partis. Et l'éminent succès de la politique adoptée à l'égard de cet État nous montre de quelle manière on peut obtenir un résultat identique dans le BasCanada. (Le rapport Durham, Montréal, Éditions Sainte-Marie, CSM 13-14, (1969): 126-127, traduction par Denis Bertrand et Albert Desbiens).

Tel l'oeuf de Colomb... et ne serait-ce qu'à ce titre, le travail de Hamel gagnerait certes à être davantage diffusé auprès de tous nos inconditionnels sécurisés par l'apparition soudainement salvatrice d'un «bon garçon» dirigeant un parti conservateur qui s'est toujours caractérisé par sa magnanimité à l'égard des francophones (lois scolaires au NouveauBrunswick et au Manitoba, Riel, Règlement XVII, etc.)

Quelques remarques maintenant - un tantinet vétillardes... - à l'égard de cet essaj. Pourquoi placer sous Appendice (p. 13) la chronologie, la bibliographie et l'index, alors que seule la première en fait partie? Pourquoi titrer globalement 1762-1900, alors que de la page 166 à 211 , 1'auteur nous fournit des textes allant de 1682 à 1761 ? Pourquoi, dans les pièces de théâtre versifiées, recommencer toujours à la ligne les parties dialoguées d'alexandrins (pp. 537-554)? Pourquoi enfin, et surtout, l'éditeur n'a-t-il pas utilisé un caractère typographique différent pour les documents cités et les écrits mêmes de Hamel, évitant de la sorte au lecteur l'inutile casse-tête de la paternité des textes (un exemple entre plusieurs autres, pp. 313-326)?

Mais comme on le voit, ces quelques oublis n'affectent nullement la valeur intrinsèque de cette somme qui, encore une fois, a le triple mérite de nous fournir un ouvrage de base comme outil de référence, une anthologie fouillée de la production louisiane francophone, et un aperçu particulièrement pertinent pour les Québécois de ce qu'est la «louisianisation».

1. Réginald Hamel, la Louisiane créole (littéraire, politique et sociale), Montréal, Leméac, 1984, ill., 2 tomes, $679 \mathrm{p}$. 